\title{
Single Actuator Alignment Control for Improved Frequency Stability of a Cavity-Based Optical Frequency Reference
}

\author{
Samuel T. Dawkins* and André N. Luiten \\ Frequency Standards and Metrology Group, School of Physics, University of Western \\ Australia, \\ 35 Stirling Hwy, Nedlands 6009 WA, Australia \\ *Corresponding author: sam@physics.uwa.edu.au
}

\begin{abstract}
We demonstrate a method of controlling the alignment of a laser beam to a Fabry-Perot resonator through synchronous detection of the misalignment arising from modulating the orientation of a single beam-steering mirror. The horizontal and vertical tilt of the mirror are modulated in quadrature to drive a circular motion of the beam orientation. A corresponding modulation of the intensity of the optical field circulating in the cavity is measured at either the reflected or transmitted port and demodulated synchronously to derive two error signals to indicate the vertical and horizontal misalignment. These signals are fed back to the beam-steering mirror to suppress fluctuations below $30 \mathrm{~Hz}$. This method avoids the complexity of monitoring off-axis cavity modes and is particularly effective in the case where unwanted pointing fluctuations are introduced by one or two elements in the optical set-up. We have applied the technique to two Fabry-Perot resonators in use as precision frequency references, delivering a result of $10 \mathrm{~dB}$ suppression of alignment fluctuations at $1 \mathrm{~Hz}$ and an improvement in frequency stability by up to a factor of 4 .

(c) 2007 Optical Society of America

OCIS codes: 120.2230, 120.3940, 220.1140.
\end{abstract}

\section{Introduction}

Fabry-Perot resonators are widely used both for their capacity to store and intensify radiation as well as to discriminate between optical frequencies. One of the technical challenges in exploiting these capabilities is the need to optimize alignment between the external optical 
beam and the modes of the cavity. For a laser beam to optimally couple power into a mode of a Fabry-Perot cavity, it must match both the frequency and spatial configuration of the mode. The latter includes the size and wavefront curvature of the beam in addition to its position and direction. One example in which controlling the alignment of a laser beam to a cavity is of importance is the use of Fabry-Perot cavities to stabilize the frequency of a laser [1-4]. Fluctuations of the alignment of the external laser with respect to the cavity modes can produce errors in the frequency locking system [5] but, more fundamentally, they can perturb the frequency of the modes of the references through fluctuations in the power coupled into the resonator. This can occur through radiation pressure $[6,7]$, but here we consider the more dominant effect of temperature changes of the components of the cavity, referred to as photothermal effects $[6,8,9]$. These fluctuations drive changes in the geometry of the cavity and thus unwanted frequency fluctuations. Our particular interest is to stabilize the power coupled into the resonator in order to address these types of effects.

We will present a technique to actively control the lateral and angular alignment of the incident laser beam in two dimensions so that it is efficiently and stably coupled into the fundamental cavity mode. We ignore issues associated with incorrect matching of beam size and wavefront curvature conditions which are assumed to be adequately met by the appropriate beam-shaping with lenses. A simple extension of this technique could be used to control these parameters as well. We also assume that the frequency of the laser beam is held at the resonant frequency of the fundamental mode using a frequency locking technique such as the Pound-Drever-Hall method [10].

As Anderson [11] has pointed out, we can model a misalignment by decomposing the incident beam shape, $U_{\text {inc }}(x)$, into a basis set of one dimensional Hermite-Gaussian modes of the cavity: $U_{0}(x), U_{1}(x), U_{2}(x) \ldots$, where $U_{0}(x)=\left(\frac{2}{\pi x_{0}^{2}}\right)^{1 / 4} \exp \left[-\left(\frac{x}{x_{0}}\right)^{2}\right]$ is the fundamental Gaussian mode with a waist size of $x_{0}$. More generally, $U_{n}(x)=\sqrt{\frac{2^{-n}}{n !}} H_{n}\left(\frac{\sqrt{2} x}{x_{0}}\right) U_{0}(x)$, is the nth-order transverse mode in the $x$-direction and $H_{n}$ is the nth order Hermite polynomial [12]. We calculate the amplitude of the nth mode via the integral:

$$
c_{n}=\int_{-\infty}^{\infty} U_{\mathrm{inc}}(x) U_{n}^{*}(x) d x
$$

where the $c_{n}$ tells us the potential excitation of each of the cavity modes and the asterisk indicates the complex conjugate. In a general resonator, the resonant frequency of the various transverse spatial modes is not degenerate and hence for a fixed input frequency it will only couple to a single member of this mode family.

For the case of a positional misalignment between the centers of the incident laser beam and the resonant mode, we can use Eq. 1 to rewrite the incident field as a decomposition in terms of the cavity modes:

$$
U_{\text {inc }}(x)=E_{\text {inc }} U_{0}\left(x-a_{x}\right)
$$




$$
=E_{\mathrm{inc}}\left[e^{-\frac{1}{2}\left(a_{x} / x_{0}\right)^{2}} U_{0}(x)+\left(a_{x} / x_{0}\right) e^{-\frac{1}{2}\left(a_{x} / x_{0}\right)^{2}} U_{1}(x)+\ldots\right]
$$

where we have defined the x-axis as the direction along which a displacement $a_{x}$ has occurred with respect to a cavity mode that has a waist of $x_{0}$ in that dimension (as depicted in Fig. 1a), while $E_{\text {inc }}$ is the amplitude of the incident field. The fraction of incident power that would enter the fundamental mode $U_{0}(x)$, given the appropriate frequency tuning, is just the integral of the squared magnitude of the relevant part of the field:

$$
\begin{aligned}
P_{0}\left(a_{x}\right) & =\int_{-\infty}^{\infty}\left|E_{\mathrm{inc}} e^{-\frac{1}{2}\left(a_{x} / x_{0}\right)^{2}} U_{0}(x)\right|^{2} d x \\
& =E_{\mathrm{inc}}^{2} e^{-\left(a_{x} / x_{0}\right)^{2}} \\
& \simeq E_{\mathrm{inc}}^{2}\left[1-\left(a_{x} / x_{0}\right)^{2}\right],
\end{aligned}
$$

where the final line is approximated to second order in $a_{x}$. Similarly, the power of the field aligned with the first transverse mode, $U_{1}(x)$, is given by

$$
\begin{aligned}
P_{1}\left(a_{x}\right) & =E_{\mathrm{inc}}^{2}\left(a_{x} / x_{0}\right)^{2} e^{-\left(a_{x} / x_{0}\right)^{2}} \\
& \simeq E_{\mathrm{inc}}^{2}\left(a_{x} / x_{0}\right)^{2}
\end{aligned}
$$

For an angular misalignment, we can write

$$
U_{\mathrm{inc}}(x) \simeq E_{\mathrm{inc}}\left[e^{-\frac{1}{2}\left(\alpha_{x} / \alpha_{0}\right)^{2}} U_{0}(x)+i\left(\alpha_{x} / \alpha_{0}\right) e^{-\frac{1}{2}\left(\alpha_{x} / \alpha_{0}\right)^{2}} U_{1}(x)+\ldots\right],
$$

where $\alpha_{x}$ is a horizontal tilt (depicted in Fig. 1b) and we define $\alpha_{0} \equiv \frac{\lambda}{\pi x_{0}}$, where $\lambda$ is the wavelength of the light. The potential excitation of the first transverse mode (with appropriate frequency tuning) is then

$$
\begin{aligned}
P_{0}\left(\alpha_{x}\right) & \simeq \int_{-\infty}^{\infty}\left|E_{\mathrm{inc}} e^{-\frac{1}{2}\left(\alpha_{x} / \alpha_{0}\right)^{2}} U_{0}(x)\right|^{2} d x \\
& \simeq E_{\mathrm{inc}}^{2}\left[1-\left(\alpha_{x} / \alpha_{0}\right)^{2}\right] . \\
P_{1}\left(\alpha_{x}\right) & \simeq \int_{-\infty}^{\infty}\left|E_{\mathrm{inc}} i\left(\alpha_{x} / \alpha_{0}\right) e^{-\frac{1}{2}\left(\alpha_{x} / \alpha_{0}\right)^{2}} U_{1}(x)\right|^{2} d x \\
& \simeq E_{\mathrm{inc}}^{2}\left(\alpha_{x} / \alpha_{0}\right)^{2} .
\end{aligned}
$$

Equations 3, 4, 6 and 7 show that a small lateral or angular misalignment effectively transfers some of the optical power available to couple to the fundamental mode into the first transverse mode. This means that a detection of power coupled into the first transverse mode would imply a misalignment. However, in most applications the laser field is not resonant with these transverse modes as its frequency has been actively controlled to be resonant with the fundamental Gaussian mode of a non-degenerate cavity.

Sampas and Anderson [13] overcame this problem by frequency modulating the incident light field to shift some optical power to the frequencies corresponding to off-axis modes. By 


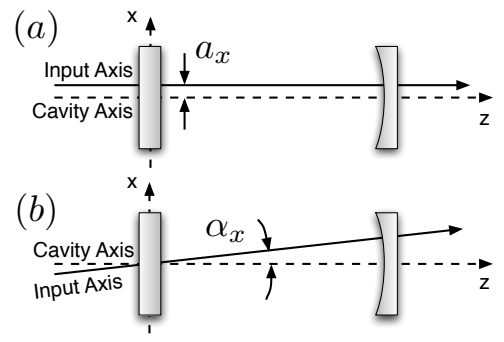

Fig. 1. We consider (a) the lateral and (b) angular misalignment in the horizontal $x$-direction of a hemispherical optical cavity.

monitoring the amplitude of the light transmitted through these transverse modes they were able to monitor and reduce the degree of misalignment. Another way to detect the presence of a misaligned (and therefore non-resonant) field is to measure the spatial asymmetry resulting from the interference of its reflection from the Fabry-Perot cavity with the leaked resonant field [14-17]. This approach avoids the need for modulation, but requires multiple wave-front sensors and complicated detection electronics to extract the relevant control signals.

An alternative to measuring the increase of power in the transverse modes [13] is to detect the reduction of power in the fundamental mode (as per Equations 3 and 6). Kawabe et al. [18] did this with a suspended Fabry-Perot in a gravitational wave detector by laterally modulating the Fabry-Perot mirrors themselves. By measuring the modulation in the power coupled into the fundamental mode, this technique detects the misalignment in terms of the lateral position of each mirror and corrects it. Similarly, Logan et al. [19] controlled the alignment in a displacement-measuring retroreflector-based interferometer by modulating the angle of the launcher optics to keep the beam measuring along the correct optical path. A similar approach is also used for the commercial automation of the alignment of optical fiber, in which the vertical and horizontal position of a fiber is modulated by a piezoelectric mount in order to maximize the transmission through the fiber [20].

In this paper we propose and implement a simple technique for the alignment of a beam to the mode of a fixed Fabry-Perot cavity; we modulate the orientation of a single beamsteering mirror and detect its effect on the power coupled into the resonator. We derive a measure of the beam's alignment to the cavity in terms of the mirror's orientation, and use this signal to suppress a combination of lateral and angular misalignment. We note that this approach does not give the possibility to distinguish between lateral and angular misalignments separately; however, there is frequently a strong correlation between the angular and positional noise (arising because a single optical element causes both) and in this case a judicious positioning of the actuating mirror can effectively suppress the effects of both types 


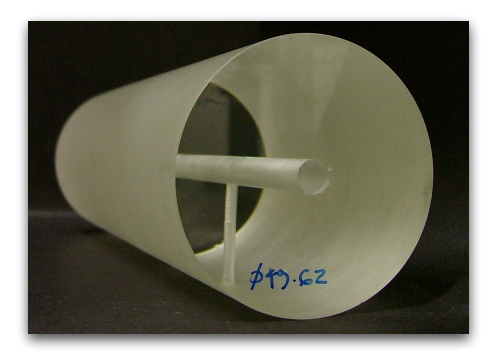

Fig. 2. The sapphire spacer of $O F R_{2}$ (The spacer of $O F R_{1}$ has equivalent dimensions).

of alignment fluctuations. The modulation is applied to two axes of the beam-steering mirror at the same frequency but with an intentional $90^{\circ}$ phase shift to produce a circular motion. By using synchronous two-phase detection, we send feedback to the actuator to correct for angular and positional noise, which causes fluctuations of circulating power in the cavity. Through the optimization and stabilization of the circulating power, power-driven temperature fluctuations of the cavity are suppressed together with the frequency fluctuations of the resonator originating from these effects. The novelty of our approach is its simplicity when compared to alternative techniques. In addition, the technique actively locks to the cavity mode and not to just some fixed point on the optical table.

\section{Optical Resonators}

In this paper, we control the beam alignment of a precision optical frequency reference for the purpose of improving its frequency stability. We have implemented this control algorithm on two separate references in order that we can measure the improvement in frequency fluctuations resulting from this control. The two optical frequency references are very similar and will be referred to as $O F R_{1}$ and $O F R_{2}$. At the heart of each reference is a FabryPerot resonator comprised of two high-finesse mirrors separated by a $15 \mathrm{~cm}$-long cylindrical sapphire spacer (pictured in Fig. 2). Both resonators are hemispherical, having a flat front mirror and a concave back mirror with a radius of curvature of $500 \mathrm{~mm}$ for $O F R_{1}$ and $222 \mathrm{~mm}$ for $O F R_{2}$. The mirrors are coated with a low-loss dielectric to produce a finesse of 1380 for $O F R_{1}$ and 5650 for $O F R_{2}$. In both cavities the mirrors have been spring-loaded onto the spacers using metallic clamps. Both references have been mounted on low thermal conductivity rigid posts to support them in the centre of a thermally controlled vacuum chamber.

These chambers have two stages of temperature-controlled shielding (shown schematically for $O F R_{1}$ in Fig. 3). The intent is that thermal fluctuations at each stage are passively sup- 


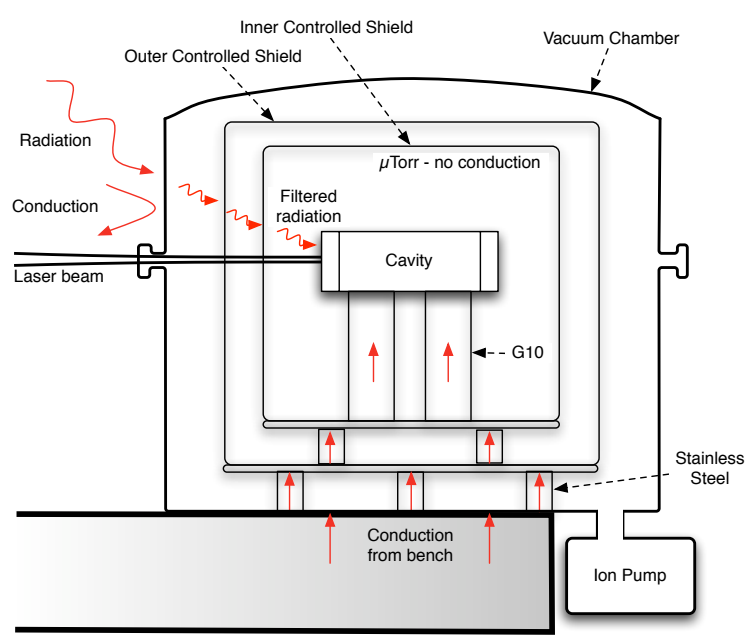

Fig. 3. Both Fabry-Perot resonators are kept at a constant temperature using two-stages of thermal shielding.

pressed due to the thermal filtering produced by the insulating vacuum between stages. The cavity spacer features a central bore to ensure that the resonant field is not affected by the optical loss of the sapphire crystal. We use an ion pump to hold the vacuum below $10^{-5}$ Torr to avoid perturbations to the resonant frequency caused by refractive index fluctuations associated with the presence of air.

We direct $1064 \mathrm{~nm}$ radiation from a Nd:YAG laser source to each resonator (Lightwave Electronics 124 for $O F R_{1}$ and Innolight Prometheus 20NE for $O F R_{2}$ ) and manually align the beams to the cavity axis to maximize the power coupled into the fundamental axial mode. Each laser is frequency modulated with its internal piezoelectric crystal (at a rate of $211 \mathrm{kHz}$ for $O F R_{1}$ and $487.3 \mathrm{kHz}$ for $O F R_{2}$ ) [21] in order to produce a locking signal as per the Pound-Drever-Hall scheme. This signal is fed back via a loop-filter to the same piezoelectric crystal to keep each laser's frequency locked to the axial mode of its respective resonator. The frequency control loop bandwidth is approximately $15 \mathrm{kHz}$ with two stages of integration to provide additional gain below about $1 \mathrm{kHz}$. The combined residual noise of these control loops is at the level of $0.4 \mathrm{~Hz} / \sqrt{\mathrm{Hz}}$.

\section{Sensitivity to Alignment}

The principal objective of this work is to reduce frequency fluctuations in our resonators by reducing alignment fluctuations. Frequency fluctuations of the optical references were monitored by mutual comparison through heterodyning on a photodiode (see Fig. 5), which gives rise to a signal at the difference of the two optical frequencies of approximately $1 \mathrm{GHz}$, 


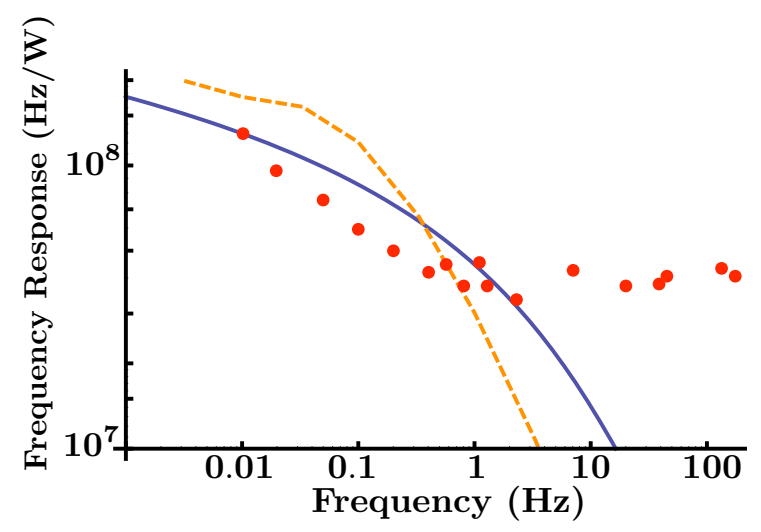

Fig. 4. The sensitivity of $O F R_{1}$ to fluctuations of incident power, measured by modulating the optical power with a variable optical attenuator (dots). The solid line is the photothermal response calculated using a theoretical model [6] and the dashed line is a calculation using finite element analysis.

convenient for measurement with a frequency counter.

Using this signal, the cavity resonance frequency was found to be sensitive to alignment, which we believe is due to a combination of a fast heating of the cavity mirrors plus a slow heating of the cavity spacer, both in response to variations of the in-coupled power. The sensitivity of the cavity to fluctuations of in-coupled power alone was separately measured by placing an LCD variable optical attenuator (MeadowLark Model B1020) in the beam path. This allowed the power incident on the cavity to be modulated without affecting the alignment in order to measure the corresponding cavity frequency response as shown in Fig. 4. We confirmed that the low frequency part of the curve was due to the photothermal response of the cavity by modeling with a time-dependent finite element analysis package (FlexPDE), which is shown in Fig. 4 together with a model devised by Cerdonio et al. [6] and measured experimentally by de Rosa et al. [8]. For both of these models, we have used the known mirror parameters and material properties, but estimated the mirror absorption, which is unknown. The deviation of our experimental results from the model at higher frequencies is most likely due to a direct power sensitivity of the frequency locking servo resulting in a spurious locking signal that creates a flat power sensitivity.

In order to characterize the alignment sensitivity, we included two mirror actuators in the beam's path to the cavity. The first is a Cambridge Technologies 603X mirror positioning assembly which employs two small orthogonal mirrors actuated by coil scanners. The second is a Thorlabs KC1-PZ piezoelectric mirror mount which can tilt a single mirror in two axes. 


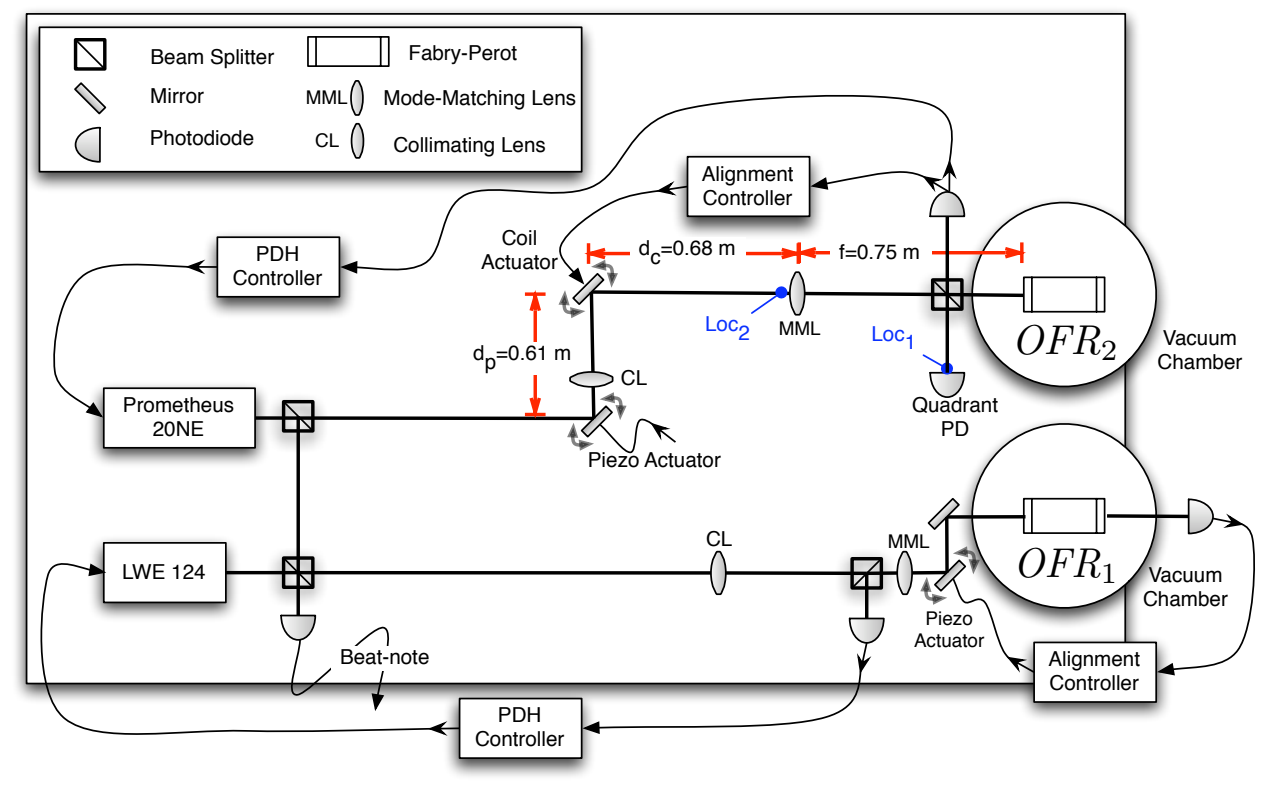

Fig. 5. Schematic of the optical layout.

The principle function of these mirror assemblies is to change the angle of the beam according to a signal supplied to the unit. In general, there is also a small change to the position since the beam is usually not positioned exactly on the axis of rotation of each mirror. For the purposes of the simple analysis presented below we have ignored this effect. For $O F R_{2}$, we have both a coil actuator and a piezoelectric actuator in the beam path, which allows us to apply pure positional and angular modulations at the input of the cavity (see Fig. 5). For $O F R_{1}$, we use only a single piezoelectric mirror mount.

Modulation of the direction of the beam at some point in its path will, in general, produce both an angular and positional modulation at the cavity input. Mathematically we can show this with the ABCD matrix approach [12]: a horizontal angular deflection at the actuator, $\phi_{A}$, results in a combination of transverse and angular misalignments ( $x^{\prime}$ and $\phi^{\prime}$ respectively) after passing through an arbitrarily complicated series of optics (as represented by the arbitrary ray matrix):

$$
\left(\begin{array}{l}
x^{\prime} \\
\phi^{\prime}
\end{array}\right)=\left(\begin{array}{ll}
A & B \\
C & D
\end{array}\right)\left(\begin{array}{c}
0 \\
\phi_{A}
\end{array}\right)=\left(\begin{array}{c}
B \phi_{A} \\
D \phi_{A}
\end{array}\right) .
$$

In our case, the coil actuator is positioned where the beam is collimated, before it passes to a mode-matching lens that has been carefully positioned to be a focal length away from the front mirror of the cavity. Therefore, a modulation at the coil actuator of $\phi_{C}$ causes both 
positional and angular modulations at the cavity according to:

$$
\left(\begin{array}{l}
a_{x} \\
\alpha_{x}
\end{array}\right)=\left(\begin{array}{ll}
1 & f \\
0 & 1
\end{array}\right)\left(\begin{array}{cc}
1 & 0 \\
-\frac{1}{f} & 1
\end{array}\right)\left(\begin{array}{cc}
1 & d_{C} \\
0 & 1
\end{array}\right)\left(\begin{array}{c}
0 \\
\phi_{C}
\end{array}\right)=\left(\begin{array}{c}
f \phi_{C} \\
\left(1-\frac{d_{C}}{f}\right) \phi_{C}
\end{array}\right),
$$

where $d_{C}$ is the distance from the coil actuator to the mode-matching lens of focal length $f$, and we have expressed the result in terms of the previously defined cavity misalignment parameters $a_{x}$ and $\alpha_{x}$.

By inducing an intentional alignment modulation in this way, we were able to observe the resulting modulation of the cavity frequency in the beat-note. For ease of comparison with the above analysis, we measured sensitivity to both a pure lateral misalignment and a pure angular misalignment. This was achieved by using a second piezoelectric actuator (as shown in Fig. 5) in conjunction with the original coil actuator to perturb only the one degree of freedom. Mathematically, we model this by including an extra term in Eq. 9 for the contribution of the piezoelectric actuator, which deflects the beam by $\phi_{P}$ at a distance of $d_{P}$ from the coil actuator:

$$
\begin{aligned}
\left(\begin{array}{l}
a_{x} \\
\alpha_{x}
\end{array}\right) & =\left(\begin{array}{c}
f \phi_{C} \\
\left(1-\frac{d_{C}}{f}\right) \phi_{C}
\end{array}\right)+\left(\begin{array}{ll}
1 & f \\
0 & 1
\end{array}\right)\left(\begin{array}{cc}
1 & 0 \\
-\frac{1}{f} & 1
\end{array}\right)\left(\begin{array}{cc}
1 & d_{C} \\
0 & 1
\end{array}\right)\left(\begin{array}{cc}
1 & d_{P} \\
0 & 1
\end{array}\right)\left(\begin{array}{c}
0 \\
\phi_{P}
\end{array}\right) \\
& =\left(\begin{array}{c}
f\left(\phi_{C}+\phi_{P}\right) \\
\left(1-\frac{d_{C}}{f}\right)\left(\phi_{C}+\phi_{P}\right)-\frac{d_{p}}{f} \phi_{P}
\end{array}\right),
\end{aligned}
$$

where we have ignored the effect of the collimating lens shown in Fig. 5 because it is very close to the piezoelectric actuator. This reveals that we get a pure angular deviation when $\phi_{P}=\phi_{C}$ and a pure lateral deviation when $\phi_{P}=\frac{d_{C}-f}{d_{C}-f+d_{P}} \phi_{C}$.

In practice, we found this balance experimentally by monitoring the modulation on a position-sensitive detector (PSD). For a pure angular modulation, we direct a sample of the incident beam to the PSD positioned at the same optical distance as the front mirror of the cavity (indicated as $L_{0}$ in Fig. 5). The PSD is insensitive to beam angle and so we balance the actuators to null the signal, leaving only pure angular modulation. The amount of pure angular modulation resulting is estimated by re-positioning the PSD to just before the mode-matching lens (indicated as $\mathrm{Loc}_{2}$ in Fig. 5). At this location the pure angular motion at the front mirror of the cavity corresponds to the pure positional motion measured by the PSD.

Alternatively, for achieving pure positional modulation, we adjust the relative magnitude of the actuator modulations to minimize the modulation seen by the the quadrant photodiode at $\mathrm{LC}_{2}$. The level of pure positional modulation at the cavity can be measured subsequently at $L o c_{1}$. For each case, the frequency deviation induced by an alignment modulation at $1 \mathrm{~Hz}$ was measured on the beat-note giving sensitivities of $5.8 \mathrm{MHz} / \mathrm{m}$ and $2.4 \mathrm{MHz} / \mathrm{rad}$ in 

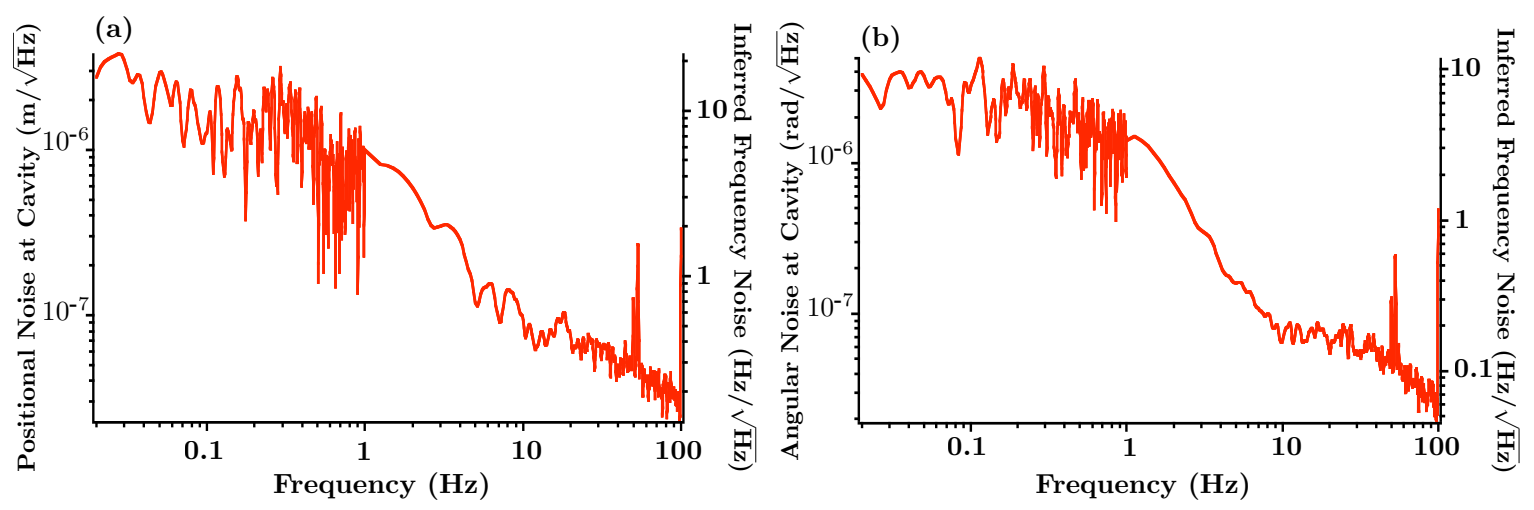

Fig. 6. The noise spectrum at the front mirror of the $O F R_{2}$ cavity of (a) vertical position inferred from the positional noise measured at $L o c_{1}$ and (b) vertical tilt inferred from the positional noise measured at $L o c_{2}$.

the vertical axis. A separate measurement indicated that the sensitivity in the horizontal axis was similar, and that the frequency dependence is relatively flat between $1-30 \mathrm{~Hz}$, as is expected from Fig. 4.

The environmentally-induced fluctuations in position and angle of the beam at the front mirror of the cavity were inferred from the measurements with the PSD at $L o c_{1}$ and $L o c_{2}$ and are presented in Fig. 6. In addition, we have also used the senstivities above to calculate the inferred frequency fluctuations resulting from these environmental peturbations and shown this on the right axis of each of these figures. It is seen that the positional fluctuations are responsible for the majority of the frequency fluctuations (by a ratio of about 2-3 to 1). What is also noted is that the ratio of the positional to angular noise is relatively constant across the curves at the level of about $1 \pm 0.4 \mathrm{~m}$. In a previous measurement we have seen a very similar curve for optical length fluctuations caused by the movement of air in a typical laboratory environment [22]. This suggests that the origin of the low frequency part of the curve is air fluctuations while the high frequency part is pointing noise of the laser.

\section{Alignment Control}

\section{A. Multiplexing Alignment into Coupled Power}

In order to create a sensor for misalignment we intentionally modulate the mirror position and search for synchronous power fluctuations. By using the result in Eq. 9 with Eqs. 3 and 6 , we see that the angle of the actuator, $\phi_{A}$, transforms into the fractional power in the fundamental mode by

$$
P_{a}\left(\phi_{A}\right)=1-\left(\frac{f}{x_{0}}\right)^{2} \phi_{A}^{2}
$$




$$
P_{\alpha}\left(\phi_{A}\right)=\left(\frac{\pi x_{0}}{\lambda}\right)^{2}\left(1-\frac{d}{f}\right)^{2} \phi_{A}^{2}
$$

In our experiment, the laser frequency is held at the resonance frequency of the fundamental mode, which is not degenerate with the higher order transverse modes. Therefore, the power absorbed by the cavity, and that transmitted through the cavity, both depend upon the degree of alignment to the fundamental mode and on the incident power. For the strongest alignment signal, one should monitor the variations in power at the cavity port with the highest average power. In our case this is the transmitted port for $O F R_{1}$ and the reflected port for $\mathrm{OFR}_{2}$. We note that the use of the reflected port increases the potential for intensity noise arising from technical issues such as parasitic interferometers caused by unwanted reflections in the beam path. On $O F R_{2}$, such an effect, related to the angle of a beam splitter, was found to give rise to an order of magnitude increase of intensity noise, enough to compromise the action of the alignment control. However, by careful adjustment of the beam splitter angle, this effect was mitigated.

The relationships in Eq. 11 show that we could attempt to maximize the transmitted power (or equivalently minimize the reflected power) through optimal alignment of the actuator. However, direct detection of the DC-level of transmitted or reflected power is also sensitive to other sources of power fluctuations such as those from the laser and other optical elements in the beam path, or simply variations in the optical sensitivity of the photodiode. To circumvent these problems, we modulate the alignment of the beam and synchronously monitor the resulting modulation of the transmitted or reflected power.

For a small intentional angular modulation of the actuator, $\delta \phi$, the effect on the power in the circulating power is given by:

$$
\delta P \simeq \frac{\partial P_{a}}{\partial \phi_{A}} \delta \phi+\frac{\partial P_{\alpha}}{\partial \phi_{A}} \delta \phi
$$

which combined with Eq. 11 shows the resulting effect of the actuator's angle on the power coupled into the cavity:

$$
\delta P \simeq-\left[2 \frac{f^{2}}{x_{0}^{2}}+2\left(\frac{\pi x_{0}}{\lambda}\right)^{2}\left(1-\frac{d}{f}\right)^{2}\right] \phi_{A} \delta \phi \sim-3 \times 10^{7} \phi_{A} \delta \phi,
$$

where we have substituted the parameters relevant to $O F R_{2}: d=0.68 \mathrm{~m}, f=0.75 \mathrm{~m}, x_{0}=$ $188 \mu \mathrm{m}$ and $\lambda=1.064 \mu \mathrm{m}$. This shows that resulting modulation of the power, $\delta P$ is directly proportional to both the actuator's average misalignment, $\phi_{A}$, as well as the modulation level, $\delta \phi$.

Eq. 12 demonstrates that any deflection from the optimal angle of the laser beam at the location of the actuator, including those caused by environmental influences, will manifest as a modulation of the circulating power of the cavity. We note, however, that pure positional 


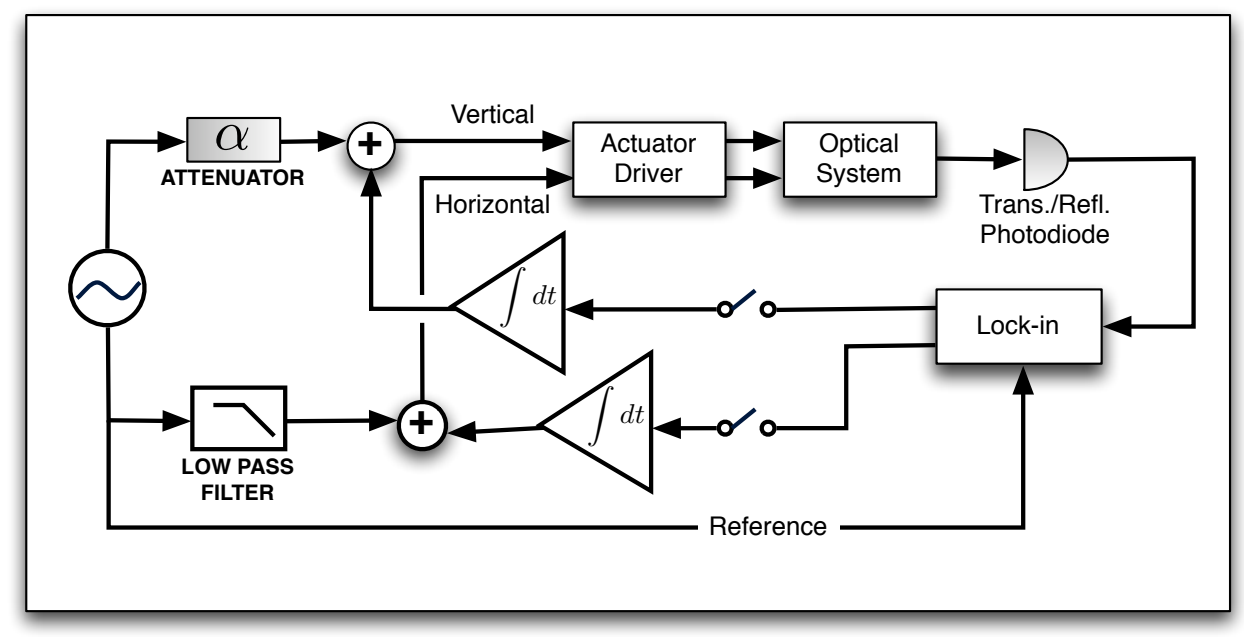

Fig. 7. Schematic of the alignment control system.

deflections at the location of the actuator will not contribute, and will thus be undetected. To detect these fluctuations, we would require a second actuator at a conjugate location where they manifest as angular fluctuations. However, a single actuator positioned where the angular fluctuations dominate the positional fluctuations can still work effectively, as demonstrated in this article.

\section{B. Control Signal Detection}

In order to generate a control signal for our servo, we detect the amount of modulation with a dual-phase lock-in amplifier (Femto LIA-MVD-200-H). The horizontal and vertical orientation of the mirror are modulated in quadrature. The phase of the lock-in amplifier is adjusted so that each channel is maximally sensitive to either the horizontal or vertical misalignment. For $O F R_{2}$, we used a galvanic actuator with a modulation frequency of $6.11 \mathrm{kHz}$. For the slower piezoelectric actuator used on $O F R_{1}$, it was necessary to carefully choose a modulation frequency $(207.5 \mathrm{~Hz})$ between mechanical resonances in order that the modulation frequency was well outside the required control bandwidth. The signal produced by the lock-in amplifiers is integrated and then summed to the original modulation signal, as shown in the schematic of the control system in Fig. 7. 

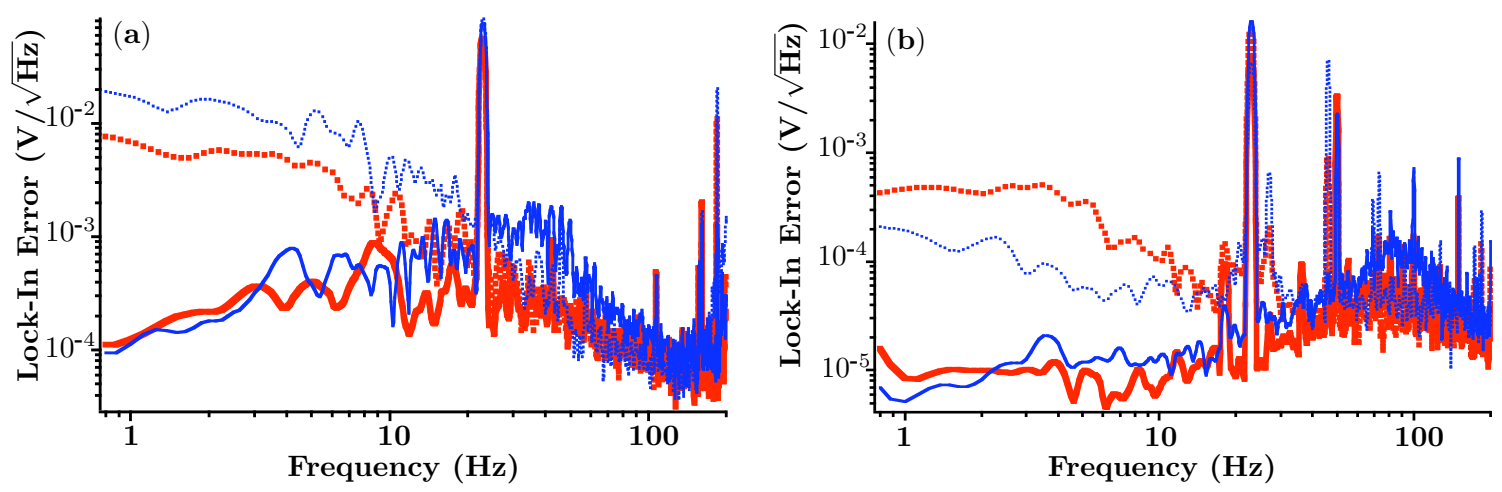

Fig. 8. The spectral density of the in-loop error signal when the alignment lock is off (dotted lines) and on (solid lines) for the horizontal (thick red lines) and vertical (thin blue lines) directions of (a) $O F R_{1}$ and (b) $O F R_{2}$.

\section{Results}

\section{A. Lock Performance}

Figure 8 shows a comparison of closed loop and open loop alignment fluctuations in terms of the error signal derived from the transmitted power of $O F R_{1}$ and the reflected power of $O F R_{2}$. We see a control bandwidth of about $17 \mathrm{~Hz}$ and $20 \mathrm{~Hz}$ for the horizontal and vertical axes of $O F R_{1}$ and $18 \mathrm{~Hz}$ and $30 \mathrm{~Hz}$ for those of $O F R_{2}$. There is no need for higher bandwidths since above approximately $20 \mathrm{~Hz}$ the power fluctuations in the input signal is dominated by amplitude noise derived from the laser, and therefore cannot be effectively controlled by alignment adjustments.

We verified on $\mathrm{OFR}_{2}$ that not only were the power fluctuations in the cavity lessened, but that this was associated with a reduction in the pointing fluctuations of the beam by measuring the beam position with the position sensitive detector. The spectrum of the outof-loop noise shown in Figure 9 shows that in our target frequency range of $0.1 \mathrm{~Hz}$ to $10 \mathrm{~Hz}$ the alignment instability has been attenuated by at least $10 \mathrm{~dB}$ at $1 \mathrm{~Hz}$. The noise floor of the alignment system prevents the system attenuating any further. This noise arises from the electronic noise floor of the photodiode.

\section{B. Frequency Stability}

The efficacy of the alignment control was measured by comparing the power spectral density of the laser frequency fluctuations between when the alignment lock was operating to when it was switched off (see Fig. 5). This frequency-noise spectrum was measured by phase-locking a low-noise microwave synthesizer to the difference frequency between $O F R_{1}$ and $O F R_{2}$ and 


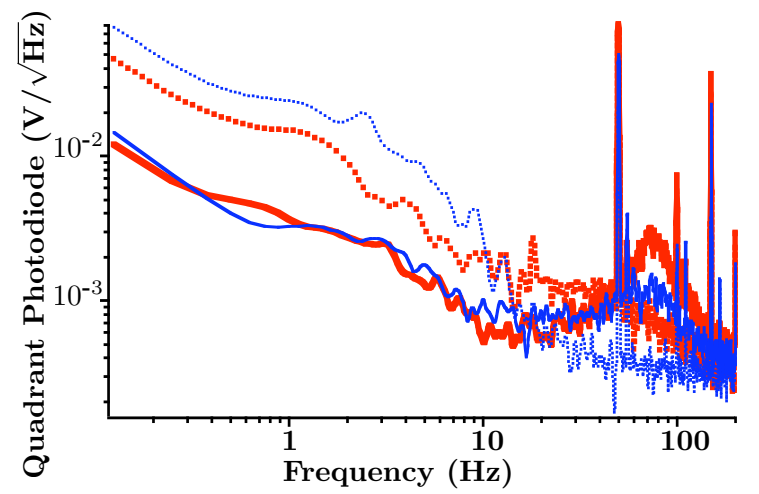

Fig. 9. The spectral density of the out-of-loop error signal as measured by the quadrant photodiode in $L o c_{1}$ when the alignment lock is off (dotted lines) and on (solid lines) for the horizontal (thick red lines) and vertical (thin blue lines) directions of $O F R_{2}$.

delivering the control signal from the phase-locked loop to an FFT spectrum analyzer, as depicted in Fig. 10. Figure 11a shows the power spectral density with the alignment servos off and on, which shows a broad-band reduction of frequency fluctuations by a factor of 2 . We note that by inspection of Eq. 12, the power fluctuations are affected by both the actuator's average misalignment $\left(\phi_{A}\right)$ and the level of misalignment fluctuations $(\delta \phi)$. Suppression of the former there reduces the power fluctuation over a broad frequency range, while suppression of the latter occurs only within the control bandwidth.

For a clearer indication of the improvement, we also delivered the beat-note to a frequency counter and determined the square root Allan variance according to common practice. In order to show that the measurements were in approximate agreement (although the timescales do not overlap), we converted the power spectral density result from Fig. 11a into the corresponding variance and show the two in Fig. 11b. We note that the frequency counter was in timed-arming mode and therefore the measurement is not strictly the Allan variance but the Triangle variance discussed in [23].

We note that the improvement in frequency stability is not as substantial as might have been predicted given the reduction in alignment fluctuations (see Fig. 9). We have made vibration measurements with a seismometer that indicate that part of this is due to seismic and acoustic vibrations perturbing the cavity frequency. Our apparatus is mounted on an air-suspended optical table, which helps reduce seismic noise only above the table's resonance frequency at $2.5 \mathrm{~Hz}$ (visible as the small hump on the solid line in Fig. 11). Fig. 11 shows the minimum instability achieved was $2 \mathrm{~Hz} / \sqrt{\mathrm{Hz}}$ at $12 \mathrm{~Hz}$ on the spectrum and $3 \times 10^{-14}$ at $0.4 \mathrm{~s}$ on the square root variance. 


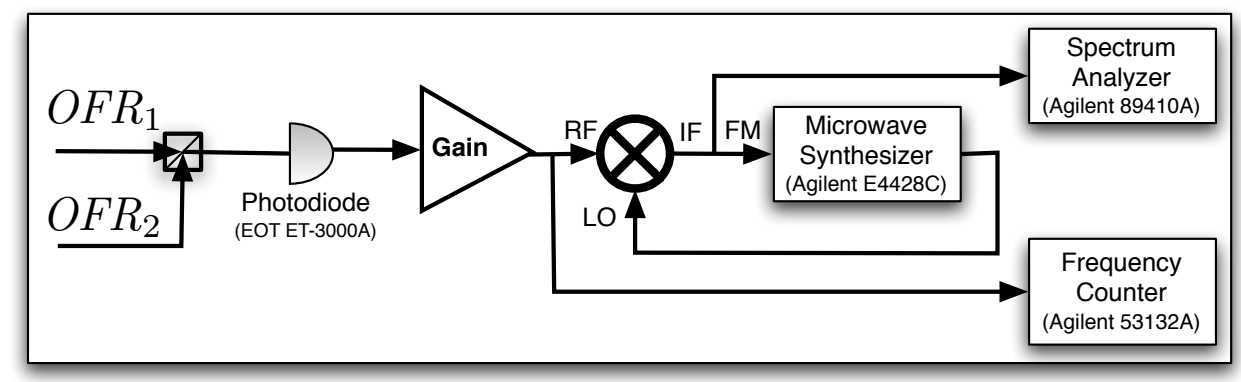

Fig. 10. The frequency stability was assessed using both a spectrum analyzer with a phase-lock-loop and a frequency counter.
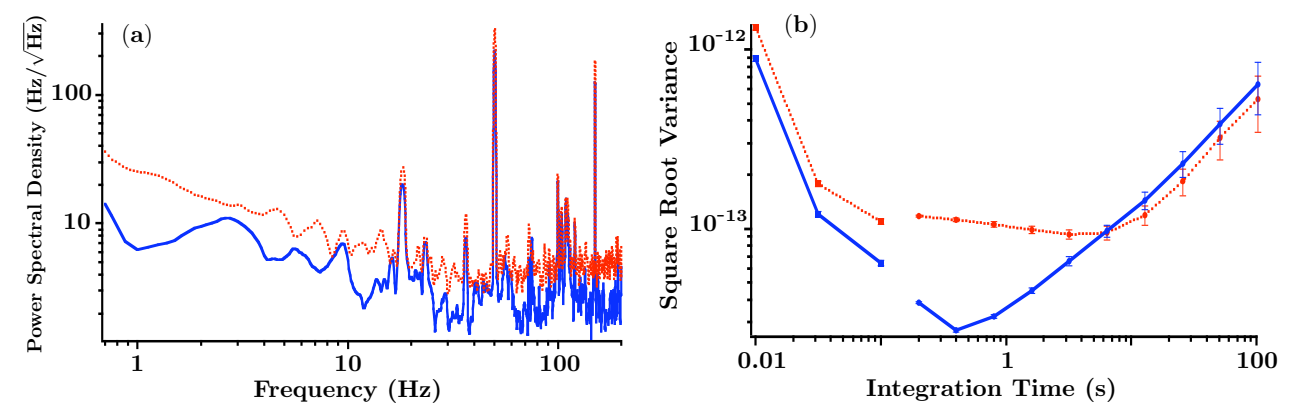

Fig. 11. The frequency noise with the alignment control off (dotted lines) and on (solid lines) as measured on the beat-note with (a) a spectrum analyzer and (right-most traces in b) a frequency counter. Also shown (left-most traces in b) are the square-root variances calculated from the power spectral density. As is standard practice for comparisons between two independent frequency references, a factor of $\sqrt{2}$ has been removed from these curves. 


\section{Conclusion}

We have demonstrated a simple and convenient modulation-based alignment control scheme of frequency-locked Fabry-Perot cavities. The optical layout requires only the inclusion of a single alignment actuator and a photodiode at one of the resonator's ports (which is perhaps already present for frequency locking). Some simple electronics allows one to close the loop and therefore attenuate the fluctuations. In our case, we controlled with only one actuator and chose a location in the optical beam path that maximized the potential for suppression of alignment fluctuations, and in doing so frequency fluctuations. The vertical and horizontal alignment fluctuations were suppressed by $10 \mathrm{~dB}$ at $1 \mathrm{~Hz}$ on two separate cavities, and a factor of 4 improvement in the frequency stability at $0.4 \mathrm{~s}$ was observed.

\section{Acknowledgments}

The authors would like to thank John Winterflood for the use of his quadrant photodiode and Tim Le Souef and Frank van Kann for their help constructing $O F R_{1}$, along with the Physics Workshop. This work was funded by the Australian Research Council.

\section{References}

1. M. Notcutt, L.-S. Ma, J. Ye, and J. Hall, "Simple and compact 1-hz laser system via an improved mounting configuration of a reference cavity," Opt. Lett. 30, 1815 - 17 (2005/07/15).

2. S. A. Webster, M. Oxborrow, and P. Gill, "Subhertz-linewidth nd:yag laser," Optics Letters 29, 1497-1499 (2004).

3. M. Eichenseer, A. Y. Nevsky, J. von Zanthier, and H. Walther, "A nd:yag laser with short term frequency stability at the hertz level," Quantum Electronics Conference, 2003. EQEC '03. European pp. 241- (2003).

4. B. C. Young, F. C. Cruz, W. M. Itano, and J. C. Bergquist, "Visible Lasers with Subhertz Linewidths," Physical Review Letters 82, 3799-3802 (1999).

5. C. Salomon, D. Hils, and J. L. Hall, "Laser stabilization at the millihertz level," Journal of the Optical Society of America B Optical Physics 5, 1576-1587 (1988).

6. M. Cerdonio, L. Conti, A. Heidmann, and M. Pinard, "Thermoelastic effects at low temperatures and quantum limits in displacement measurements," Physical Review D 63, 082003-+ (2001).

7. B. C. Buchler, M. B. Gray, D. A. Shaddock, T. C. Ralph, and D. E. McClelland, "Suppression of classicand quantum radiation pressure noise by electro-optic feedback," Optics Letters 24, 259-261 (1999). 
8. M. de Rosa, L. Conti, M. Cerdonio, M. Pinard, and F. Marin, "Experimental Measurement of the Dynamic Photothermal Effect in Fabry-Perot Cavities for Gravitational Wave Detectors," Physical Review Letters 89, 237402-+ (2002).

9. E. D. Black, I. S. Grudinin, S. R. Rao, and K. G. Libbrecht, "Enhanced photothermal displacement spectroscopy for thin-film characterization using a Fabry-Perot resonator," Journal of Applied Physics 95, 7655-7659 (2004).

10. R. W. P. Drever, J. L. Hall, F. V. Kowalski, J. Hough, G. M. Ford, A. J. Munley, and H. Ward, "Laser phase and frequency stabilization using an optical resonator," Applied Physics B: Lasers and Optics 31, 97-105 (1983).

11. D. Z. Anderson, "Alignment of resonant optical cavities," Applied Optics 23, 2944-2949 (1984).

12. A. E. Siegman, Lasers (Published by University Science Books, ISBN 0-935702-11-3, 1986).

13. N. M. Sampas and D. Z. Anderson, "Stabilization of laser beam alignment to an optical resonator by heterodyne detection of off-axis modes," Appl. Opt. 29, 394-403 (1990).

14. E. Morrison, B. J. Meers, D. I. Robertson, and H. Ward, "Experimental demonstration of an automatic alignment system for optical interferometers," Applied Optics 33, 50375040 (1994).

15. N. Mavalvala, D. Sigg, and D. Shoemaker, "Experimental test of an alignment-sensing scheme for a gravitational-wave interferometer," Applied Optics 37, 7743-7746 (1998).

16. J. H. Chow, D. Rabeling, M. B. Gray, D. E. McClelland, and G. de Vine, "Spot size and guoy phase invariant telescope for auto-alignment of resonant cavities," Classical and Quantum Gravity 21, S909-S914 (2004).

17. B. J. J. Slagmolen, M. Barton, C. Mow-Lowry, G. de Vine, D. S. Rabeling, J. H. Chow, A. Romann, C. Zhao, M. B. Gray, and D. E. McClelland, "Alignment locking to suspended Fabry-Perot cavity," General Relativity and Gravitation 37, 1601-1608 (2005).

18. K. Kawabe, N. Mio, and K. Tsubono, "Automatic alignment-control system for a suspended Fabry-Perot cavity," Appl. Opt. 33, 5498-5505 (1994).

19. J. E. Logan, P. G. Halverson, M. W. Regehr, and R. E. Spero, "Automatic alignment of a displacement-measuring heterodyne interferometer," Applied Optics 41, 4314 (2002).

20. Melles Griot Inc., Catalogue X, Melles Griot Inc., 2051 Palomar Airport Road, Carlsbad, CA 92011, USA (2005).

21. G. Cantatore, F. Della Valle, E. Milotti, P. Pace, E. Zavattini, E. Polacco, F. Perrone, C. Rizzo, G. Zavattini, and G. Ruoso, "Frequency locking of a nd:yag laser using the laser itself as the optical phase modulator," Review of Scientific Instruments 66, 2785-2787 (1995).

22. R. P. Kovacich, "The precision of modern phase coherent optical to microwave frequency 
chains," Ph.D. thesis, The University of Western Australia, 35 Stirling Hwy, Nedlands W.A. 6009, Australia (2000).

23. S. T. Dawkins, J. J. McFerran, and A. N. Luiten, "Considerations on the measurement of the stability of oscillators with frequency counters," IEEE Transactions on Ultrasonics, Ferroelectrics, and Frequency Control 56 (2007). 